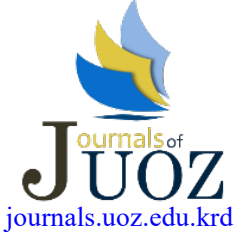

Soience Journal of University of Zakho

Vol. 8, No. 3, pp. 112-117, September-2020

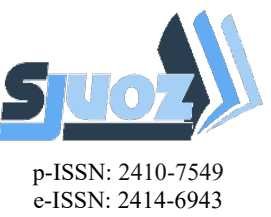

\section{AN IMAGE DATASET CONSTRUCTION FOR FLOWER RECOGNITION USING CONVOLUTIONAL NEURAL NETWORK}

\author{
Saman M. Omer ${ }^{\text {a }}$, Rayan M. Hasan ${ }^{\text {a }}$, Banaz N. Anwer ${ }^{\mathrm{a}}$ \\ ${ }^{\text {a }}$ Department of Software Engineering, Faculty of Engineering, Koya University, Koya KOY45, Kurdistan Region - F.R. Iraq - \\ (saman.muhammad@koyauniversity.org ; rayan.855787@gmail.com ; banaznihad99@gmail.com)
}

Received: Jul., 2020 / Accepted: Aug., 2020 / Published: Sep.,2020

https://doi.org/10.25271/sjuoz.2020.8.3.747

\begin{abstract}
:
Classifying flowers is a difficult activity because of the wide variety of flower species that have similar form. In this paper, a deep learning model for extracting features and classifying different flower types or species developed by using a popular method called Convolutional Neural Network (CNN). The identification system has been evaluated on a new dataset that has been designed in this work that collected flowers from Kurdistan. The dataset contains 1300 images of different flowers, 1040 images (\%80) of which used for training purpose and $260(\% 20)$ images used for test purpose, categorized into 26 classes. In addition, Various number of layers, activation function and pooling schemes were implemented to improve the classification rates. The recognition rate of proposed $\mathrm{CNN}$ model is $94.61 \%$.
\end{abstract}

KEYWORDS: Plant Flower Recognition, Flower Recognition, Flower Identification System, Flower Classification, Convolutional Neural Network, Deep Learning.

\section{INTRODUCTION}

Plants are an essential part of life on Earth, providing us with breathable oxygen, food, medication and etc. Moreover, plants help to control the climate change, providing food for insects and other animals and give a natural way to regulate flooding. Nowadays, plant classification and safety become more essential in the fields of botany, horticulture, forestry, environmental and medical sciences and numerous related disciplines (Asaf, et al., 2017). The study of this area is crucial to help in recognizing new or unusual plant species in order to progress the drug industry, balance the ecosystem as well as the agricultural productivity and sustainability (Cope, Corney, Clark, Remagnino, \& Wilkin, 2012). Existing plant identification systems rely on personal knowledge, which has not provided the feasible classification and protection method. An automated system is required to identify and classify plants. Automatic plant image identification has been focused on in both computer vision and botanical taxonomy, and it is the best solution for bridging the botanical taxonomic gap (Sun, Liu, Wang, \& Zhang, 2017). The issue of identification in plant is faced with a number of challenges because of differences in shape, color, texture and venation within a plant family (Asaf, et al., 2017). There are many ways and efforts that have been conducted for researchers and botanist to recognize a plant such as, identification and extracting local features from its flowers, leaves, stem, fruit, and root (Sun, Liu, Wang, \& Zhang, 2017).

Computer Vision approaches have been suggested for automatic analysis of botanical organs. Flower is an organ of plant which is one of the basic feature that can be used in identification of plants. Some studies have been made on flower recognition to classify the species of plants due to its exclusive features. In the past years, ordinarily people used a guide book and relevant websites to get information about flower types, and ( (Silva, Pinho, Lopes, Nogueira, \& Silveira, 2011)) developed an Interactive Dichotomous Key (IDK) to help botany for identifying leaf plant that includes a questioner related to the subject which is available on website. All those techniques caused wasting a lot of time and made people bored without getting enough an accurate information. In these cases, automatic recognition of flower is important.

Machine Learning is a popular technology that is capable for finding particular solutions to problems in different fields such as image processing.

Deep learning as a new area of machine learning has become well-known in classification. It is efficient for learning and processing from the composite input data. In the fields of images, sounds, text and motion, it has been applied effectively (Du \& Shanker, 2009). Recently, deep learning algorithms are proposed to resolve the problem with distinguished achievement compare with algorithms that had been used before 2006, and beating the state-of- the-art in assured areas (Du \& Shanker, 2009) (Bengio, Learning Deep Architectures for AI , 2009). Classical image processing methods depends on hand-designed features that are chosen manually, but the deep learning techniques have the capability to overcome the drawback of traditional algorithms and would be suitable for big data analysis that are automatically select features. This enables deep learning methods to obtain better outcomes than the previous techniques used in (Liua, et al., 2017) (LeCun, Bengio, \& Hinton, 2015) (Asaf, et al., 2017).

Convolutional Neural Network (CNN) is a special category of Neural Networks. It has a significant role in Image Classification and Segmentation, Object Detection, Video Processing, Natural

* Corresponding author

This is an open access under a CC BY-NC-SA 4.0 license (https://creativecommons.org/licenses/by-nc-sa/4.0/) 
Language Processing, and Speech Recognition (Khan, Sohail, Zahoora, \& Qureshi, 2019). Deep CNN architectures are becoming very common due to great results in nearly all image recognition problems (Liua, et al., 2017).

The process of an accurate flower recognition is very challenging because of the inter classes sample similarity and the dissimilarity of intra class samples (Gurnani, Mavani, Gajjar, \& Khandhediya, 2017) (S.M.Mukane \& J.A.Kendule, 2013 ), in addition to the wide variety of flower groups which is over 220,000 (Cope et al., 2012). Furthermore, there are some more issues lead to a perplexity over classes and make the errand of bloom classification more challenging such as background of images that is usually contain similar objects. In a real environment, pictures of blossoms are frequently taken in common open-air scenes where lighting condition changes with the climate and time, the distance to the flower, and there are a lot more variation in viewpoint of flower images (S.M.Mukane \& J.A.Kendule, 2013).

In this paper, $\mathrm{CNN}$ is proposed to extract features and classify the plant flower images. Experiments have been applied on KRD26 Flower dataset that has been designed in this study which contains flower images that grow in the Kurdistan region habitat.

The objectives in this work could be summarized as:

- Create a structured plant flower images dataset which was not addressed in the previous studies. Flower photos have been taken in Kurdistan Region. It can be further used in other research problems.

- To develop a method that can be reliably used to classify plants through their flower images.

- $\quad$ Applying the state of the art deep learning model (CNN) to improve accuracy results.

The rest of this paper is organized as follows: in section 2 various related research techniques with their working procedure has been discussed. Section 3 addressed the proposed methodology, experiments setup is described in section 4, while the performance evaluation and result experiments are discussed in section 5. Finally, the research work is concluded in section 6 .

\section{RELATED WORK}

Recently, many studies have been worked on automatic plant recognition in different fields such as leaf, root, stem and fruit, especially in the field of flower on various datasets that vary in their data sizes (number of samples) and conditions. Normally, computer vision tasks required intelligent segmentation of an image, to understand what is in the image and enable easier analysis of each part. The first step in most of the flower classification processes is the image segmentation. It is a crucial process in computer vision and involves dividing a visual input into segments to simplify image analysis. The segments represent objects or parts of objects, and comprise sets of pixels. Many techniques have been used for flower segmentation. For instance, ChanVese Active Contour model was used for segmenting flowers from the rest of the image to simplify and enhance the features extraction process in (Almogdady, Manaseer, \& Hiary, 2018). Flower's region only is segmented from the background in (S.M.Mukane \& J.A.Kendule, 2013) using a semi-automated threshold-based segmentation algorithm. Albadarneh \& Ahmad deployed region growing segmentation method in a manner that keeps the flower region fully segmented with the pre-processing steps (Albadarneh \& Ahmad, 2017). The segmentation process can be also involved for computer vision model based on deep learning. For example, (Hiary, Saadeh, Saadeh, \& Yaqub, 2018) developed deep learning-based method to segment and classify flower images. Fully Convolutional Network (FCN) framework method was developed for segmenting the flower region that localises the flower by detecting the minimum bounding box around the object. In addition, Prasad et al. proposed a model based on CNN for implementing classification directly on flower image to eliminate the typical job of segmentation (Prasad, et al., 2018). The segmentation processes in this paper has not done automatically but rather the images have been pre-processed and segmented as will be presented in section 3.1.

Feature plays an important role in the area of pattern recognition in images. Color, shape and texture are the features that have been used as characteristic descriptors, in order to differentiate between flower object (foreground), and other irrelevant objects (background). Many hand crafted features were extracted by researchers in classifying the objects using traditional methods. For instance, in (C.Bhanuprakash, GK, Karegowda, \& Ramesh, 2016) proposed a method for flower classification using neural network algorithm based on Gray Level Co-occurrence Matrix (GLCM) texture features. Mukane and Kendule presented the effect of texture feature using neural network for flower recognition with GLCM and discrete wavelet transform (DWT) (S.M.Mukane \& J.A.Kendule, 2013). A combination set of features extracted in (Almogdady, Manaseer, \& Hiary, 2018) to classify flower images such as Invariant Moment (IM) as a shape descriptor, GLCM as a texture descriptor and Hue, Saturation and Value (HSV) as a color descriptor. The testing was initiated on 7 features with single feature and multiple feature and found that an improvement for recognition accuracy from 39.15\% into $81.19 \%$ for multiple feature. Furthermore, in (Albadarneh \& Ahmad, 2017) designed an automated system to recognize flower spices. In the study, six features such as RGB and HSV as Color features, GLCM and Wavelet transform as textural features, HOG and Scale Invariant Feature Transform (SIFT) as shape features had been extracted and combined together. The experimental results indicate that combining features together obtaining higher recognition accuracy than using the same feature individually. In recent studies, deep learning especially $\mathrm{CNN}$ has gained popularity in computer vision applications including plant flower recognition. In the case of applying $\mathrm{CNN}$, extraction of flower features and the classification process are combined in the same model. The powerful learning ability of deep $\mathrm{CNN}$ is primarily due to the use of multiple feature extraction stages that can automatically learn representations from the data and avoid the procedure of complicated feature extraction. In this paper, we have proposed a CNN model to extract the learned feature for flower image recognition.

Classification is a supervised learning approach in which the computer program learns from the input data and then uses this learning to classify new observations. Machine learning-based methods have been used for flower recognition in some studies. For instance, (S.M.Mukane \& J.A.Kendule, 2013) used Artificial Neural Network (ANN) for flower recognition and achieved accuracy of $87 \%$. Almogdady et. al, used Back-Propagation ANN as classifier for flower recognition with accuracy $80 \%$ (Almogdady, Manaseer, \& Hiary, 2018). Stochastic Gradient Descent (SGD) classifier approach has been used for flower classification task in (Albadarneh \& Ahmad, 2017). On the other hand, deep learning techniques, especially CNN- based, is also proposed for flower classification task. CNN has recently gained a lot of interest in solving numerous learning problems due to greater accuracy compared with classical methods. Hiary et al, developed a CNN method to accurately classify the different flower classes (Hiary, Saadeh, Saadeh, \& Yaqub, 2018). In another work, CNN model has been proposed for implementing flower classification directly from flower images on Oxford 102 and KL University Flower Dataset (KLUFD) of 30 classes (designed for the study) sum out of 9500 flower images with 132 classes in (Prasad, et al., 2018). Deep CNN is suitable for giving 
solutions to complex problems with huge quantity of data. For this purpose, in (Bengio, Deep Learning of Representations for Unsupervised and Transfer Learning, 2012) fine-tuning network is adopted based on the concept of transfer learning. The fine-tuning strategy verified to be a good solution to transfer learned recognition with the highest possible accuracy according to (Reyes, Caicedo, \& Camargo, 2015) study. Here, deep CNN was proposed for plant classification and back-propagation algorithm used to learn the network. ILSVRC 2012 dataset used to pre-train the proposed $\mathrm{CNN}$ and fine-tuning. An average precision accuracy of the system is $48.6 \%$ for 7 different views such as entire plant, branch, flower, fruit, leaf, stem and leaf scans. Additionally, Lee et. al, employed a CNN model to incorporate species and organ features for plant classification system. The result of the proposed high-level fusion architecture is $68.9 \%$, it has lower classification accuracy compared to the fine-tuned VGG-16 net which is $71.2 \%$ with augmentation. The network is trained with back-propagation, using SGD in (Lee, Chang, Chan, \& Remagnino, 2016). Furthermore, (FatihahSahidan, Juha, Mohammad, \& Ibrahim, 2019) proposed a CNN model for plant identification based on flower and leaf images. The study that utilized leaf images become more accurate with accuracy $98 \%$ result compared with flower images with $74 \%$ or both of flower and leaf together with $85 \%$ accuracy.

In previous studies, various datasets have been used for flower classification tasks. Some of those datasets are available online for researchers to be used on the research such as Oxford17, Oxford102, Zou-Nagy and Flower Recognition dataset. On the other hand, some researchers have designed a new dataset then used for classification purposes. For instance, (Albadarneh \& Ahmad, 2017) designed a Jordanian Flower Dataset. FatihahSahidan et. al, designed KL University Flower Dataset (KLUFD) (FatihahSahidan, Juha, Mohammad, \& Ibrahim, 2019).

For this purpose, the proposed work designed a new flower dataset called KRD26, all images are collected in Kurdistan region and used for flower classification task as will be presented in section 3.1.

\section{RESEARCH METHODOLOGY}

\subsection{KRD26 Flower Dataset}

There are a few publicly available flower datasets that have been used by researchers such as Oxfor 17 and Oxford 102 . The Kurdistan (KRD26) flower dataset has been designed in this study for the same purpose. The data is collected from natural scene in Kurdistan region. It consists of 26 species of ornamental flowers. Information about Kurdistan region flowers species was collected by visiting a local botanist. In the proposed system for collecting images we went to the Erbil, Koya, Darbandixan, and Soran district and took the images. Figure 1 illustrates sample images from KRD26 dataset. Some species in our dataset were given its accurate names based on botanist expertise, some others are named based on internet information. The dataset consists of 1,300 images of flowers that belongs to 26 classes, each class contains 50 different flower images. The dataset is split into the training set (40 images per class), and test set (10 images per class). Thus, 1040 images $(\% 80)$ for training, and 260 images $(\% 20)$ for testing sets.

The KRD26 dataset consists of images that have been taken from top angle. Other flower images taken from a level angle.
Flower images may vary in sizes and orientations, with some deformed images and shadows due to an order of magnitude, distances and illumination changes. By looking at Figure 1, we can see that a robust method is highly required in order to perform the flower recognition since the flower images from the same classes may have different shapes.

A white background can highlight the object and help the system to better recognition. Most of researchers apply saliencysegmentation-based approaches to select the Region-Of-Interest (ROI) on flower images. The dataset has been designed and the images have been captured using white paper as a background as shown in Figure 2. Then the dataset updated using Photoshop software to add the white background and show only flowers on the image without any noises. We had more similarity between the images in the normal dataset, then a similarity was very low after the dataset has been updated. The hardware devices that have been used for capturing the images of the dataset are (Nikon D3100, full HD, 14.2 MP), (Iphone Xmax mobile camera, full $\mathrm{HD}, 12 \mathrm{MP}$ ), and (Canon EOS 1100D is a 12.2-megapixel camera). All samples are collected during 2 months (December 2019 and January 2020).

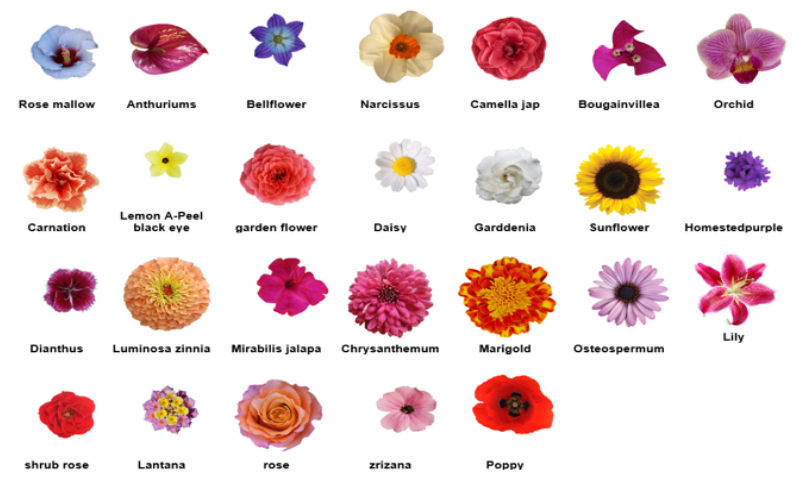

Figure 1. Dataset Image spices

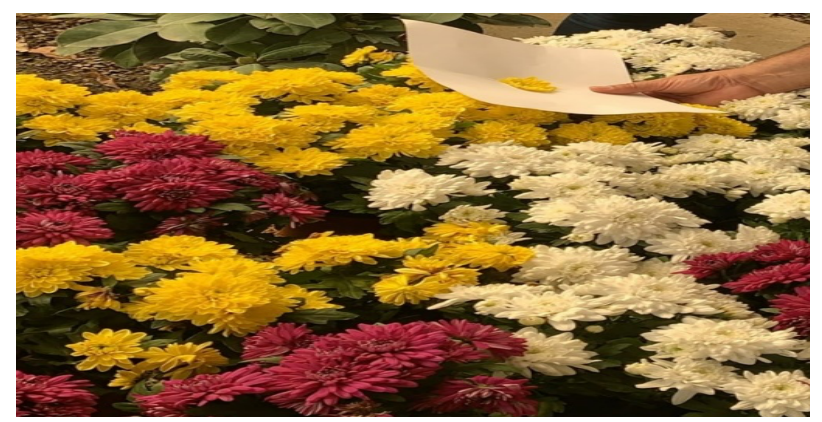

Figure 2. Capturing dataset samples

\subsection{Proposed System Architecture}

CNN model is designed in this work. The model includes four convolutional layers, two max pooling layers, one dense and SoftMax output layer.

The convolutional layer filter window size is $5 \times 5$ for all layers. The number of filters are $128,64,32$ and 32 for the layers respectively, with a stride of 1 and it is followed by a ReLU activation function from layer 1 to 4 respectively to extract features hierarchically with (max pooling). Fully connected layers used for classification task with a SoftMax output layer see figure 3. 


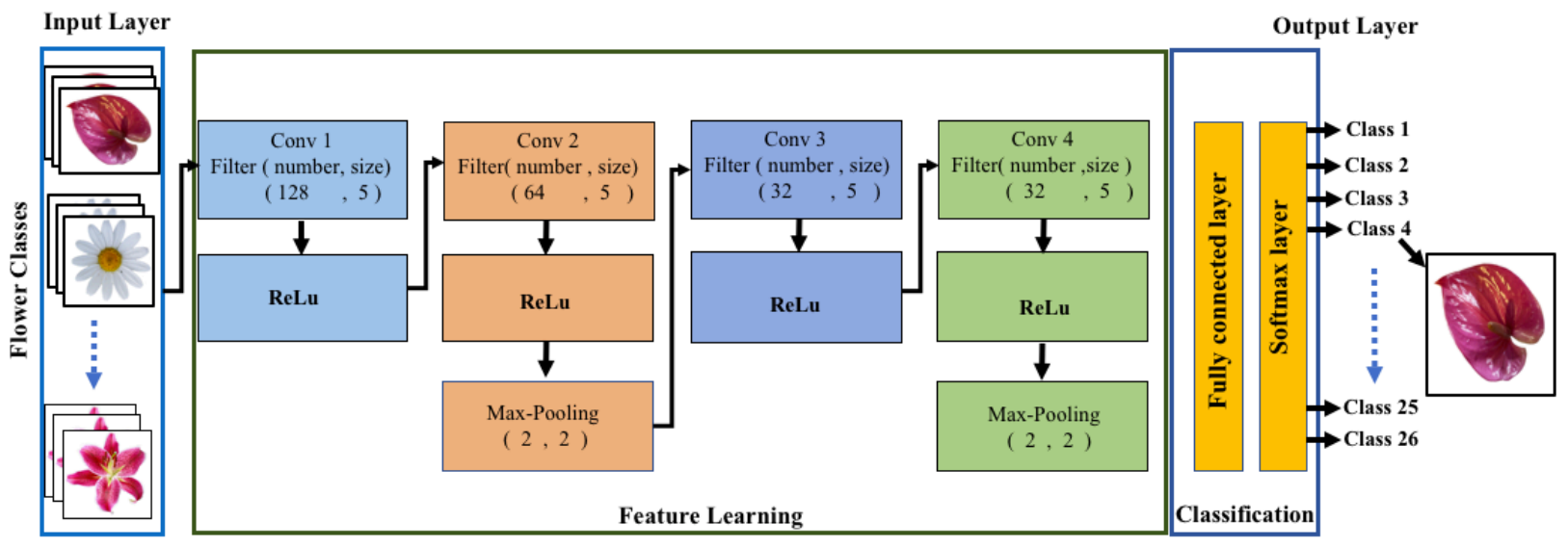

Figure 3. Proposed CNN Architecture

Low-level features such as edges are normally extracts in the first and second convolution layers while high-level features such as the shape extracts in the third and fourth convolution layers (FatihahSahidan, Juha, Mohammad, \& Ibrahim, 2019). Designing new architectures and attaining enhanced performance depends on the CNN components arrangement (Khan, Sohail, Zahoora, \& Qureshi, 2019).

The flower images of size $1000 \times 1000$ are taken as input to the system. For this CNN model, the images are resized to a smaller size, $64 \times 64$ pixels. Using the original resolution is make the procedure challenging due to the limitation of memory of the device used for this training.

In this paper, four different modified models have been adopted based on CNN architecture, which are described in the following:

Model 1: The model includes two convolutional layers followed by activation function with stride 1, two max pooling layers, one dense and SoftMax output layer. This means that pooling layer followed after first convolution layers and second convolution layers as well. The model has been tested 6 times by modifying number of filters, window sizes of convolution layer and activation functions.

Model 2: The model includes three convolutional layers followed by activation function with stride 1 , two max pooling layers, one dense and SoftMax output layer. The first max pooling layer comes next to the second convolution layer, while the second max pooling comes next to the third convolution layer. The model has been tested 3 times by modifying the number of filters, window sizes of convolution layer, pooling layer size and batch-size.

Model 3: The model includes four convolutional layers followed by activation function with stride 1, two max pooling layers, one dense and SoftMax output layer. The first max pooling layer comes next to the second convolution layer, while the second max pooling comes next to the fourth convolution layer. The model has been tested 4 times by modifying input image size, number of filters, window sizes of convolution layer and number of epochs.

Model 4: The model includes five convolutional layers followed by activation function with stride 1, three max pooling layers with stride 1, one dense and SoftMax output layer. The first max pooling layer comes next to the second convolution layer, the second max pooling comes next to the fourth convolution layer, while the third max pooling followed after fifth convolutional layer. The model has been tested 2 times by modifying number of filters, window sizes of convolutional layer and number of input image size.

\section{EXPERIMENTS SETUP}

The proposed system has been run and implemented on python and Anaconda's Spyder 3.3.6 as an IDE, and tested on an Intel (R) Core i5 CPU, 2.40GHz, 8GB Memory, x64-based processor. The main aim of this study is to correctly classify the flower image from flower dataset that has been created and flowers collected in Kurdistan Region. CNN model has been used to extract and classify the flower database. The dataset consists of 1300 flower images that belongs to 26 spices. The dataset was randomly split into $80 \%$ training data, and $20 \%$ testing data. The achieved recognition accuracy rate is $94.61 \%$.

\section{RESULT AND DISCUSSION}

Several experiments have been done to determine the best combination of parameters to produce the highest accuracy for flower recognition. Experiments were conducted by changing the number of layers, the values of the parameters in the convolutional layer, activation function, pooling layer and input image size. CNN model has been tested and results of the experiments are showed in Table 1. The table include in the first column the number of models that have been produced as explained in section 3.2, the second column shows the size of input image, while the third column presents the number of testes have been applied on KRD26 dataset, the fourth column indicate the number of filters and window size of convolutional layers where the first number in the square bracket represents the filter number while the second number represents the convolutional window size. The fifth column shows max-pooling layer, the six column shows activation function layer. Batch size has been indicated in column 7 , which is define the number of samples to work through before updating the internal model parameters. The number of epoch is shown in column 8 . And finally, the recognition accuracy has been shown in column 9 .

Table 1 realize that the accuracy result from testing CNN model on Flower dataset has been affected by using different number of layers, filter sizes, convolution window sizes, pooling layers, activation function. By looking at table 1, we can see that the highest accuracy result has been achieved with four convolutional layers which is $\mathbf{9 4 . 6 1 \%}$. Recall is used as a measure.

Similarities has been investigated among those species. It has been found that one spices could be closest to some other species, such as testing examples from Camella jap species against Carnation, rose and rose mallow, gave $10 \%$ to each of them. In addition, testing examples from Mirabilis jalapa specie against Camella Jap and Dianthus, gave $10 \%$ to each of them. The closest species with their recognition accuracy ranges are recorded in Table 2 . 
Table 1. Experimental Results on Parameters for Basic CNN

\begin{tabular}{|c|c|c|c|c|c|c|c|c|}
\hline $\begin{array}{l}\frac{3}{8} \\
\frac{8}{8}\end{array}$ & 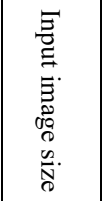 & 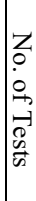 & 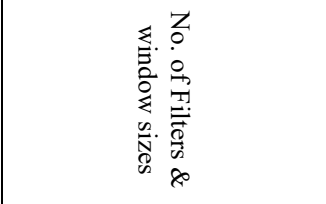 & 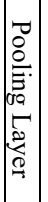 & 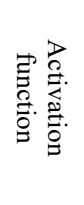 & 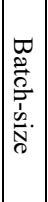 & $\begin{array}{l}8 \\
8 \\
8 \\
\\
\end{array}$ & త̂ \\
\hline \multirow{6}{*}{1} & \multirow{6}{*}{$64 \times 64$} & 1 & {$[64,5],[64,5]$} & \multirow{4}{*}{2} & \multirow{4}{*}{ ReLu } & \multirow{4}{*}{32} & \multirow{4}{*}{18} & 86.54 \\
\hline & & 2 & {$[64,3],[64,3]$} & & & & & 86.53 \\
\hline & & 3 & {$[64,9],[64,9]$} & & & & & 85.34 \\
\hline & & 4 & {$[32,5],[32,5]$} & & & & & 89.23 \\
\hline & & 5 & {$[64,5],[64,5]$} & 2 & Elu & 32 & 18 & 29.62 \\
\hline & & 6 & {$[64,5],[64,5]$} & 2 & Linear & 32 & 18 & 66.54 \\
\hline \multirow{3}{*}{2} & \multirow{3}{*}{$64 \times 64$} & 1 & {$[120,3],[64,3],[32,3]$} & 2 & \multirow{3}{*}{ ReLu } & 64 & \multirow{3}{*}{18} & 77.31 \\
\hline & & 2 & {$[64,5],[32,3],[32,3]$} & 2 & & 32 & & 85.38 \\
\hline & & 3 & {$[128,9],[64,5],[32,3]$} & 4 & & 32 & & 81.54 \\
\hline \multirow{4}{*}{3} & $120 \times 120$ & 1 & {$[128,5],[64,5],[64,5],[32,5]$} & \multirow{3}{*}{2} & \multirow{3}{*}{ ReLu } & \multirow{3}{*}{32} & 18 & 85 \\
\hline & \multirow{3}{*}{$64 \times 64$} & 2 & {$[128,5],[64,5],[32,5],[32,5]$} & & & & 30 & 86.92 \\
\hline & & 3 & {$[128,5],[64,5],[32,3],[32,3]$} & & & & 35 & 93.08 \\
\hline & & 4 & {$[128,5],[64,5],[32,5],[32,5]$} & 2 & ReLu & 32 & 50 & 94.61 \\
\hline \multirow[b]{2}{*}{4} & $160 \times 160$ & 1 & $\begin{array}{c}{[128,5],[64,5],[64,5],[32,5],} \\
{[32,5]}\end{array}$ & \multirow[b]{2}{*}{2} & \multirow{2}{*}{ ReLu } & \multirow[b]{2}{*}{\begin{tabular}{l|l}
32 & 1
\end{tabular}} & \multirow[b]{2}{*}{18} & 78.46 \\
\hline & $64 \times 64$ & 2 & $\begin{array}{c}{[64,5],[64,3],[64,3][32,3],} \\
{[32,5]}\end{array}$ & & & & & 82.69 \\
\hline
\end{tabular}

Table 2. Flowers species with their closest specie

\begin{tabular}{|c|c|c|c|c|c|}
\hline $\begin{array}{c}\text { Class } \\
\text { No. }\end{array}$ & $\begin{array}{l}\text { Tested } \\
\text { Species }\end{array}$ & \multicolumn{3}{|c|}{$\begin{array}{c}\text { Recognition accuracy against closest } \\
\text { species }\end{array}$} & Similarity \\
\hline 4 & Anthuriums & \begin{tabular}{|c} 
Anthuriums \\
$\mathbf{\% 1 0 0}$
\end{tabular} & $\begin{array}{r}\text { Against } \\
\mathbf{\%} \\
\end{array}$ & $\begin{array}{l}\text { all species } \\
\text { 0 }\end{array}$ & $\begin{array}{l}\begin{array}{l}\text { Unique } \\
\text { shape }\end{array} \\
\end{array}$ \\
\hline 6 & Camella jap & $\begin{array}{c}\text { Camella } \\
\text { Jap } \\
\text { \%70 }\end{array}$ & $\begin{array}{lr}\text { Carnation } & \text { Rose } \\
& \\
\mathbf{\% 1 0} & \% 10\end{array}$ & $\begin{array}{c}\text { Rose } \\
\text { mallow } \\
\% \mathbf{1 0}\end{array}$ & texture \\
\hline 18 & $\begin{array}{c}\text { Mirabilis } \\
\text { jalapa }\end{array}$ & $\begin{array}{l}\text { Mirabilis } \\
\text { Jalapa } \\
\text { \%80 }\end{array}$ & $\begin{array}{c}\text { Camella Jap } \\
\mathbf{\% 1 0}\end{array}$ & $\begin{array}{c}\text { Dianthus } \\
\text { \%10 }\end{array}$ & $\begin{array}{c}\text { Color and } \\
\text { shape }\end{array}$ \\
\hline
\end{tabular}

Some of the flower species in the dataset have unique features; they obtained $100 \%$ recognition accuracy, i.e. it achieves $100 \%$ sensitivity. Flowers with unique features obtained $0 \%$ when testing against all other species, i.e. it has again 100\% specificity. This means that the tested instances cannot be from as any of those other species. For example, the first case in Table 2 shows that Anthuriums has unique internal shape, so tested instances of Anthuriums achieved $0 \%$ against all other species, and 100\% against Anthuriums species.

On the other hand, Table 2 shows that if the numbers of closest species increased, the recognition accuracy of the tested species reduced. For instance, Camella jap recognition accuracy decreased to $70 \%$, because it has three closest species in term of texture similarity, with recognition rate $\% 10$ for each species. In addition, Mirabilis jalapa recognition accuracy decreased to $80 \%$ because it has two closest species in term of colour and shape similarity, with recognition rate $\% 10$ for each species.

The main contribution of this work is to test our proposed model on various hyper parameters using a home designed dataset. Table 3 shows the accuracy achieved in many state of the art works. The result shows that the proposed model achieves comparable performance, despite the non-fair comparison among these methods, due to the different conditions regarding these proposed techniques.
Table 3. Previous Studies

\begin{tabular}{|c|c|c|c|c|}
\hline Publications & year & Dataset & Algorithm & Accuracy \\
\hline $\begin{array}{l}\text { Proposed } \\
\text { System }\end{array}$ & 2020 & \begin{tabular}{|c|} 
KRD26 flower \\
dataset that \\
includes 1300 \\
images belong to \\
26 classes each \\
contain 50 \\
images
\end{tabular} & CNN & $94.61 \%$ \\
\hline $\begin{array}{l}\text { (FatihahSahidan, } \\
\text { Juha, } \\
\text { Mohammad, \& } \\
\text { Ibrahim, 2019) }\end{array}$ & 2019 & \begin{tabular}{|c|} 
Flower \\
Recognition and \\
Folio Leaf Dataset
\end{tabular} & $\mathrm{CNN}$ & \begin{tabular}{|c|}
$74 \%$ for 100 \\
flower images \\
of 5 categories \\
$98 \%$ for 20 \\
leaf images of \\
5 categories \\
$85 \%$ for both \\
of them \\
\end{tabular} \\
\hline $\begin{array}{l}\text { (Hiary, Saadeh, } \\
\text { Saadeh, \& } \\
\text { Yaqub, 2018) }\end{array}$ & 2018 & $\begin{array}{l}\text { Zou-Nagy, } \\
\text { Oxford } 17 \text {, and } \\
\text { Oxford } 102 \\
\text { Dataset }\end{array}$ & $\begin{array}{l}\mathrm{CNN}, \mathrm{FCN} \\
\text { used for } \\
\text { segmentation }\end{array}$ & \begin{tabular}{|}
$96.1 \%$ for Zou \\
Nagy, \\
$91.4 \%$ for \\
Oxford 17 \\
$90.6 \%$ for \\
Oxford 102
\end{tabular} \\
\hline $\begin{array}{l}\text { (Prasad, et al., } \\
\text { 2018) }\end{array}$ & 2018 & $\begin{array}{c}\text { KLUFD and } \\
\text { Oxford102 dataset }\end{array}$ & $\mathrm{CNN}$ & $\begin{array}{l}97.78 \% \text { for } \\
\text { both of them } \\
\text { together }\end{array}$ \\
\hline $\begin{array}{l}\text { (Almogdady, } \\
\text { Manaseer, \& } \\
\text { Hiary, 2018) }\end{array}$ & 2018 & $\begin{array}{l}\text { Oxford102 } \\
\text { Dataset }\end{array}$ & BPANN & $81.19 \%$ \\
\hline $\begin{array}{c}\text { (Albadarneh \& } \\
\text { Ahmad, 2017) }\end{array}$ & 2017 & $\begin{array}{c}\text { Jordanian Flower } \\
\text { and } \\
\text { Oxford } 17 \text { Dataset }\end{array}$ & $\begin{array}{c}\text { SGD as } \\
\text { classifier, } \\
\text { segmentation } \\
\text { applied }\end{array}$ & $\begin{array}{l}92 \% \text { for } \\
\text { Jordanian } \\
\text { Flower, } \\
83.52 \% \text { for } \\
\text { Oxfoed } 17\end{array}$ \\
\hline $\begin{array}{l}\text { (C.Bhanuprakash, } \\
\text { GK, Karegowda, } \\
\text { \& Ramesh, 2016) }\end{array}$ & 2016 & $\begin{array}{c}5 \text { categories of } \\
\text { flower image in } \\
\text { three different } \\
\text { color with three } \\
\text { different angles } \\
(45,90,135) \\
\end{array}$ & $\begin{array}{c}\text { BP } \\
\text { with gradient } \\
\text { descent }\end{array}$ & $98 \%$ \\
\hline $\begin{array}{l}\text { (Lee, Chang, } \\
\text { Chan, \& } \\
\text { Remagnino, } \\
\text { 2016) } \\
\end{array}$ & 2016 & $\begin{array}{c}\text { PlantClef2015 } \\
\text { dataset }\end{array}$ & $\begin{array}{l}\text { CNN fine- } \\
\text { tuned used } \\
\text { VGG-16 }\end{array}$ & $\begin{array}{c}71.2 \% \text { for } 7 \\
\text { organs }\end{array}$ \\
\hline $\begin{array}{l}\text { (Reyes, Caicedo } \\
\text { and Camargo, } \\
\text { 2015) }\end{array}$ & 2015 & $\begin{array}{c}\text { LifeCLEF2015 } \\
\text { dataset }\end{array}$ & $\begin{array}{l}\text { CNN fine- } \\
\text { tuned }\end{array}$ & $\begin{array}{c}48 \% \text { for } 7 \\
\text { organs }\end{array}$ \\
\hline $\begin{array}{l}\text { (S.M.Mukane \& } \\
\text { J.A.Kendule, } \\
\text { 2013) }\end{array}$ & 2013 & $\begin{array}{c}5 \text { categories each } \\
\text { contain } 10 \text { flower } \\
\text { images }\end{array}$ & $\begin{array}{l}\text { ANN, MLP } \\
\text { as classifier }\end{array}$ & $87 \%$ \\
\hline
\end{tabular}

Tables 3 indicates the recognition accuracy results that have been obtained on the different flower datasets alongside recent state-of-the-art from other researcher including the proposed system.

\section{CONCLUSION}

Providing automated recognition system for classifying flower image species has many benefits to the people either in the agricultural, pharmaceutical industry, botany field or in any other fields. Many people choose such a system to identify flowers easier, it was also very challenging because flower species are 
diverse and it is very difficult to identify them since they may also be very close to each other. In this paper, the performance of CNN model has been evaluated for plant recognition using its flower on KRD26 flower dataset. Various experiments have been conducted to obtain a better recognition accuracy result. In addition, a new dataset has been constructed that include flower images in Kurdistan region. Various number of layers, activation functions and pooling schemes were implemented to improve the classification rates. The recognition accuracy rate of proposed CNN model is $94.61 \%$.

For future research, we plan to investigate other variations of the $\mathrm{CNN}$ architecture and compare with other plant datasets for more training data.

\section{AKNOWLEGMENT}

The Authors would like to thank Koya University and Raprin University for their support during the preparation of the work. Many thanks for the reviewers for their valuable comments and suggestions on the manuscript which improve the performance of the paper. Special thanks should also be given to (Dr. Abdulbasit Al-Talabani, lecturer at Koya university) for his supports and assistance during the course of the work.

\section{REFERENCES}

Albadarneh, A., \& Ahmad, A. (2017). Automated Flower Species Detection and Recognition from Digital Images. International Journal of Computer Science and Network Security, 17(4), 144-151.

Almogdady, H., Manaseer, S., \& Hiary, H. (2018). A Flower Recognition System Based On Image Processing And Neural Networks. International Journal Of Scientific \& Technology Research, 7(11), 166-173.

Asaf, Z., Siddique, M. U., Akram, M. A., Sabir, M. W., Ali, H. M., \& Akram, D. M. (n.d.). Leaf Recognition Using Deep Convolutional Neural Networks.

Bengio, Y. (2009). Learning Deep Architectures for AI (Vol. 2). Foundation and Trends in Machine Learning .

Bengio, Y. (2012). Deep Learning of Representations for Unsupervised and Transfer Learning. JMLR: Workshop and Conference Proceedings, 27, pp. 17-36.

C.Bhanuprakash, GK, P., Karegowda, A. G., \& Ramesh, C. (2016). Texture Based Flower Species Classification Using Neural Network. International Journal of Advance Foundation and Research in Computer, 3(6), $2348-4853$.

Cope, J. S., Corney, D., Clark, J. Y., Remagnino, P., \& Wilkin, P. (2012). Plant species identification using digital morphometrics: A review. Expert Systems with Applications, 39(8), 7562-7573.

Du, T., \& Shanker, V. K. (2009). Deep Learning for Natural Language Processing. ecis. Udel. Edu, 1-7.

FatihahSahidan, N., Juha, A. K., Mohammad, N., \& Ibrahim, Z (2019). Flower and leaf recognition for plant identification using convolutional neural network. Indonesian Journal of Electrical Engineering and Computer Science, 16(2), 737-743.

Gurnani, A., Mavani, V., Gajjar, V., \& Khandhediya, Y. (2017). Flower Categorization using Deep Convolutional Neural Networks. arXiv preprint arXiv:1708.03763, 4321-4324.

Hiary, H., Saadeh, H., Saadeh, M., \& Yaqub, M. (2018). Flower classification using deep convolutional neural networks. IET Computer Vision, 12(6), 855-862.

Khan, A., Sohail, A., Zahoora, U., \& Qureshi, A. S. (2019). A Survey of the Recent Architectures of Deep
Convolutional Neural Networks. arXiv preprint arXiv:1901.06032, 1-70.

LeCun, Y., Bengio, Y., \& Hinton, G. (2015). Deep learning. nature, 521(7553), 436-444.

Lee, S. H., Chang, Y. L., Chan, C. S., \& Remagnino, P. (2016). Plant Identification System based on a Convolutional Neural Network for the LifeClef 2016 Plant Classification Task. In CLEF (Working Notes), $502-$ 510

Liua, W., Wanga, Z., Liua, X., Zengb, N., Liuc, Y., \& Alsaadid, F. E. (2017). A survey of deep neural network architectures and their applications. Neurocomputing, 234, 11-26.

Prasad, M. V., Lakshmamma, B. J., Chandana, A. H., Komali, K., Manoja, M., Kumar, P. R., . . Kiran, P. S. (2018). An efficient classification of flower images with convolutional neural networks. International Journal of Engineering \& Technology, 7(1.1), 384-391.

Reyes, A. K., Caicedo, J. C., \& Camargo, J. E. (2015). Finetuning Deep Convolutional Networks for Plant Recognition. CLEF (Working Notes), (pp. 467-475).

S.M.Mukane, \& J.A.Kendule. (2013). Flower Classification Using Neural Network Based Image Processing. IOSR Journal of Electronics and Communication Engineering, 7(3), 80-85.

Silva, H., Pinho, R., Lopes, L., Nogueira, A. J., \& Silveira, P. (2011). Illustrated plant identification keys: An interactive tool to learn botany. Computers \& Education, 56(4), 969-973.

Sun, Y., Liu, Y., Wang, G., \& Zhang, H. (217). Deep Learning for Plant Identification in Natural Environment. Computational intelligence and neuroscience, 2017. 